\title{
On the Modality of Convex Polygons
}

\author{
Karl Abrahamson \\ Computer Science Department, Washington State University, \\ Pullman, WA 99164-1210, USA
}

\begin{abstract}
Under two definitions of random convex polygons, the expected modality of a random convex polygon grows without bound as the number of vertices grows. This refutes a conjecture of Aggarwal and Melville.
\end{abstract}

\section{Introduction}

Algorithms in computational geometry tend to be quite difficult, and to involve a large number of special cases. Some of the cases might be very rare. Algorithm design can be simplified by either excluding rare and difficult cases, or by designing an efficient algorithm for the common cases, and combining it with a less efficient algorithm for the rare cases. The problem is to recognize the rare cases.

For algorithms which operate on convex polygons, one useful condition on the input is unimodality. The modality of a vertex of a polygon is the number of local maxima in the sequence of distances from that vertex to the other vertices, in their natural order around the polygon. The modality of a polygon is the maximum of the modalities of its vertices. A polygon is unimodal if its modality is 1 ; otherwise, it is multimodal. Figure 1 shows polygons of modality 1 and 2.

When drawing a "random" convex polygon, it is quite common to find that the selected polygon is unimodal. In fact, Dobkin and Snyder [4] and Snyder and Tang [7] each assume that all convex polygons are unimodal in designing algorithms to find the diameter of a convex polygon. The fact that there exist multimodal convex polygons, pointed out by Avis $e t$ al. [3], thus at least initially seems counterintuitive. Aggarwal and Melville [2] conjecture that, under any reasonable definition of a random convex polygon, the probability that a random convex $n$-gon is unimodal tends to 1 as $n \rightarrow \infty$. (They do not say just what a reasonable definition would be.) If true, the conjecture would imply that multimodality is one of those rare features which it might pay to ignore. 


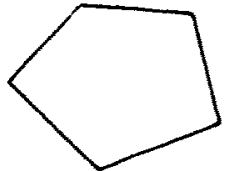

(a)

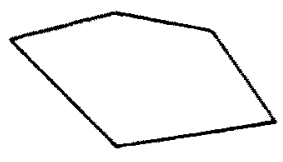

(b)

Fig. 1. Convex polygons of (a) modality 1 and (b) modality 2 .

Aggarwal and Melville [2] also give a linear-time algorithm to determine the modality of a convex polygon. Using that algorithm, an algorithm which runs in linear time, but assumes a unimodal input, can be used to construct an algorithm which is correct for any convex polygon, but runs in linear time on unimodal inputs. An example is the linear-time all-furthest-neighbors algorithm of Toussaint [8] for unimodal convex polygons. The conjecture of Aggarwal and Melville, if true, would suggest that all-furthest-neighbors for convex polygons can be solved in linear time for the expected case input. (In fact, it can be solved in linear time for any convex polygon, by the algorithm of Aggarwal et al. [1].)

This paper refutes the conjecture for two definitions of random convex polygons, and in fact shows that the probability that a random convex polygon is unimodal approaches zero quite rapidly. Thus, algorithms which perform well on unimodal convex polygons can, unfortunately, be expected to be weak. On the other hand, the expected modality is not large. It may be that algorithms can exploit the fact that an input has a small modality.

The two chosen definitions of a random convex polygon are (1) the convex hull of $n$ points drawn uniformly from a disk in the plane, and (2) the convex hull of $n$ points drawn from a two-dimensional normal distribution. The expected modality is $\Theta(\log n / \log \log n)$ in case (1) and $\Theta(\log \log n / \log \log \log n)$ in case (2). Interestingly, the expected number of vertices on the convex hull is $O\left(n^{1 / 3}\right)$

Table 1. Modalities of convex hulls of $\boldsymbol{n}$ random points.

\begin{tabular}{|c|c|c|c|c|c|c|c|}
\hline \multicolumn{4}{|c|}{ Uniform } & \multicolumn{4}{|c|}{ Normal } \\
\hline \multirow[b]{2}{*}{$n$} & \multicolumn{3}{|c|}{ Modality } & \multirow[b]{2}{*}{$n$} & \multicolumn{3}{|c|}{ Modality } \\
\hline & 1 & 2 & 3 & & 1 & 2 & 3 \\
\hline 4 & 851 & 149 & 0 & 4 & 838 & 162 & 0 \\
\hline 8 & 683 & 315 & 2 & 8 & 616 & 381 & 3 \\
\hline 16 & 589 & 405 & 6 & 16 & 489 & 504 & 7 \\
\hline 32 & 478 & 521 & 1 & 32 & 442 & 538 & 20 \\
\hline 64 & 392 & 600 & 8 & 64 & 386 & 601 & 13 \\
\hline 128 & 281 & 714 & 5 & 128 & 378 & 608 & 14 \\
\hline 256 & 204 & 791 & 5 & 256 & 362 & 618 & 20 \\
\hline 512 & 134 & 854 & 12 & 512 & 302 & 681 & 17 \\
\hline 1024 & 74 & 910 & 16 & 1024 & 278 & 706 & 16 \\
\hline
\end{tabular}


in case (1) and $O\left(\log ^{1 / 2} n\right)$ in case (2) [5], [6]. So in the cases studied here, the expected modality is $\Theta(\log h / \log \log h)$, where $h$ is the expected number of hull vertices.

The proofs of our results apply only to very large $n$. What of moderate size $n$ ? Table 1 was computed using a nonlinear additive feedback pseudorandom number generator, with 1000 samples generated for each $n$. Note that $n$ is the number of randomly selected points. The convex hulls generally had considerably fewer than $n$ vertices. For $n=1024$, the convex hulls averaged 34 vertices in the uniform case, and 11 vertices in the normal case. The table indicates that convex hulls of modality 2 (which tend to be somewhat oblong) are prevalent for moderate size point sets. No convex hulls of modality exceeding 3 were generated.

\section{Lower Bounds on Expected Modality}

This section introduces the basic techniques, and establishes lower bounds on the expected modality. The proofs are fairly crude, but relatively simple. All logarithms are base $e$, and all angles in radians.

Theorem 1. When $n$ points are drawn at random uniformly from a disk in the plane, the expected modality of their convex hull is $\Omega(\log n / \log \log n)$ and the probability that their convex hull is unimodal approaches 0 as $n \rightarrow \infty$.

Proof. Assume that $n$ is very large. Write $x \sim y$ when $\lim _{n \rightarrow \infty} y / x=1$. Assume for convenience that the disk is centered at the origin of a Cartesian coordinate system, and has unit radius. The measure of a region is the probability that a random point falls in that region, which in the present case is the area divided by $\pi$.

Let $m \sim n^{1 / 3}$ be an even integer. As will become apparent, it is no coincidence that the expected number of vertices on the convex hull of the $n$ points is $\Theta(m)$ [5], [6]. Inscribe a regular $m$-gon in the boundary of the disk, as illustrated in Fig. 2(a). Call each connected region of the disk strictly outside the $m$-gon an ear. Let $\varphi=2 \pi / m$ be the angle defined by an ear. The dimensions of an ear are approximately $\varphi \times \varphi^{2} / 8$. For future reference, note that if the disk has radius $R$, then each ear has area $\varphi R^{2} / 2-R^{2} \cos (\varphi / 2) \sin (\varphi / 2)=\varphi^{3} R^{2} / 12+O\left(\varphi^{5}\right)$. For the unit disk, each ear has area $A_{\text {ear }} \sim \varphi^{3} / 12=\Theta(1 / n)$ and measure $\sim \varphi^{3} / 12 \pi \approx 6.6 / n$.

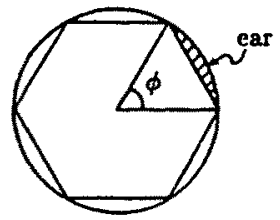

(a)

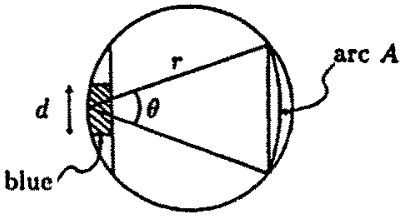

(b)

Fig. 2. The ears and the blue region. 


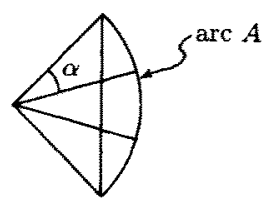

(a)

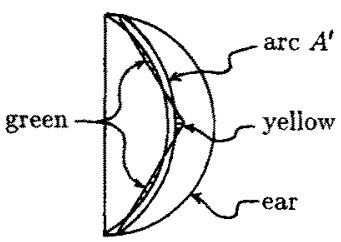

(b)

Fig. 3. (a) Angle $\alpha$, (b) the right-hand ear.

Intuitively, with fairly high likelihood points in an ear will be hull vertices which are local maxima for points in the ear on the opposite side of the disk.

We will bound the probability that the modality is $\geq k-1$ for arbitrary $k$, $1<k<m$. Choose two ears on opposite sides of the disk. Rotate the disk so that the two ears are at the left and right sides. Color a region near the center of the left-hand ear blue, as indicated in Fig. 2(b). The blue region has depth $d=\varphi / 12 k^{3}$, width $w=1-\cos (\varphi / 2)=\varphi^{2} / 8+O\left(\varphi^{4}\right)$, and area $\sim w d=\Theta\left(1 / n k^{3}\right)$.

Cut the right-hand ear with an arc $A$ of radius $r \sim 2$, centered on the point $(-1,0)$, as illustrated in Fig. 2(b). Arc $A$ defines an angle of $\theta=\varphi / 2+O\left(\varphi^{3}\right)$. Cut angle $\theta$ into $k$ equal sectors, as shown in Fig. 3(a) for $k=3$, each sector an angle of $\alpha \sim \varphi / 2 k$. In Fig. 3 angle $\alpha$ is greatly exaggerated for clarity. The plan is to bound below the probability that there is a single hull vertex in the blue region, and between each two adjacent sectors there is a single hull point which is a local maximum for the blue point. To that end, we will define green and yellow regions in the right-hand ear, as illustrated in Fig. $3(\mathrm{~b})$ for $k=2$, where the green regions will hold local minima and the yellow regions will hold local maxima for a point in the blue region.

Cut each angle $\alpha$ into six parts, as illustrated in Fig. 4(b). The region marked green is delimited by a section of arc $A$ of angle $\alpha / 3$ and its chord, and excludes its boundary. In all, there are $k$ green regions, and each has area $\sim(\alpha / 3)^{3}\left(r^{2} / 12\right)=$ $\Theta\left(\alpha^{3}\right)$.

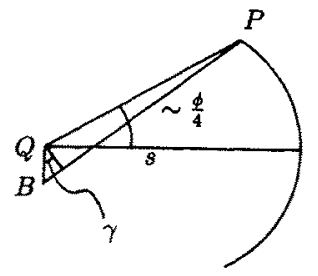

(a)

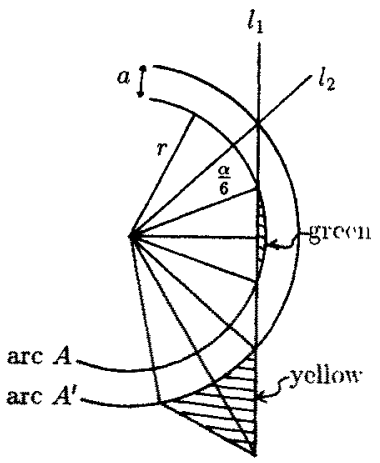

(b)

Fig. 4. (a) Proof of Claim 1, (b) yellow and green regions. 
The arc $A^{\prime}$ drawn outside $\operatorname{arc} A$ in Fig. $4(b)$ is concentric with $A$, and passes through the intersection of lines $l_{1}$ and $l_{2}$. We easily compute the separation $a$ of the two arcs from $r \cos (\alpha / 6)=(r+a) \cos (\alpha / 3)$, or $a \sim \alpha^{2} / 12 \sim k d \varphi / 4$. There are $k-1$ yellow regions, one of which is illustrated in Fig. 4(b). If the arc boundary of a yellow region is replaced by its chord, a yellow region has area

$$
\sim(r \sin (\alpha / 6))(r \sin (\alpha / 6) \tan (\alpha / 2)) \sim \alpha^{3} / 18 \text {. }
$$

The actual yellow region has area $\sim \alpha^{3} / 18-\alpha^{3} / 81=\Theta\left(\alpha^{3}\right)$. Although the diagram is badly distorted, it is easy to see that the green and yellow regions must lie entirely within the ear.

Claim 1. Let $B, G$, and $Y$ be points in the blue, green, and yellow regions, respectively. Then distance $B G$ is less than distance $B Y$.

Proof. Let $B=(x, y)$ and let $Q=(x, 0)$ be the projection of $B$ onto the $x$-axis. See Fig. 4(a). Let $s=1-x$ be the distance from $Q$ to arc $A$ along the $x$-axis. Arc $A$ is very nearly centered on $Q$, and it is easy to show that for any point $P$ on arc $A$, distance $Q P<s+O\left(\varphi^{4}\right)$. But $B P-Q P \leq(d / 2) \sin \gamma=d \varphi / 8+O\left(\varphi^{4}\right)$, where $\gamma=\varphi / 4+O\left(\varphi^{3}\right)$. Since the green regions are inside arc $A, B G \leq$ $s+d \varphi / 8+O\left(\varphi^{4}\right)$. Since the yellow regions are outside arc $A^{\prime}$, similar reasoning gives $B Y \geq s+a-d \varphi / 8-O\left(\varphi^{4}\right)$. But $a \sim k d \varphi / 4 \geq d \varphi / 2$. So $B Y-B G>$ $a-d \varphi / 4-O\left(\varphi^{4}\right)>0$.

Say that a collection of $n$ points in the disk is bad if exactly one point falls in each of the blue, green, and yellow regions, and no other points fall in either of the two ears under consideration. Notice that each of the $2 k$ points in the green, yellow, and blue regions of a bad set of points must be vertices of the convex hull. By Claim 1, each of the yellow points must be local maxima for the blue point, and the convex hull has modality at least $k-1$.

Since each ear has measure $\Theta(1 / n)$, and each of the blue, green, and yellow regions has measure $\Theta\left(\alpha^{3}\right)=\Theta\left(1 / n k^{3}\right)$, the probability that a random set of points in the disk is bad is asymptotically

$$
>\left(\begin{array}{c}
n \\
2 k
\end{array}\right)\left(\frac{c_{1}}{n k^{3}}\right)^{2 k}\left(1-\frac{c_{2}}{n}\right)^{n-2 k}>\left(\frac{c_{3}}{k^{4}}\right)^{2 k}
$$

for some positive constants $c_{1}, c_{2}$, and $c_{3}$. Let $B_{i}$ be the event that a random set of $n$ points is bad for the $i$ th pair of ears, $i=1, \ldots, m / 2$, and let $p_{k}=$ $\operatorname{Pr}\left(\bar{B}_{1} \cap \cdots \cap \bar{B}_{m / 2}\right)>\operatorname{Pr}($ the convex hull has modality $<k-1)$. Since $m \ll n$, $B_{1}, \ldots, B_{m / 2}$ are very nearly independent. So $p_{k}<\left(1-\left(c_{4} / k^{4}\right)^{2 k}\right)^{m / 2}$ for some constant $c_{4}>0$. For any fixed $k, p_{k} \rightarrow 0$ as $n \rightarrow \infty$. In fact, $p_{k} \rightarrow 0$ provided

$$
\left(\frac{m}{2}\right)\left(\frac{c_{4}}{k^{4}}\right)^{2 k} \rightarrow \infty \text { as } n \rightarrow \infty \text {. }
$$

That happens for $k \leq \log m / 9 \log \log m$, so Theorem 1 is established. 
In the case of a two-dimensional normal distribution, the expected number of points on the convex hull is only $\Theta\left(\log ^{1 / 2} n\right)$ [5], [6], so the expected modality must be lower than in the uniform case. Nevertheless, a very similar proof applies.

Theorem 2. When $n$ points are drawn from a two-dimensional normal distribution, the expected modality of their convex hull is $\Omega(\log \log n / \log \log \log n)$ and the probability that their convex hull is unimodal approaches 0 as $n \rightarrow \infty$.

Proof. Let $\varphi(x, y)=(1 / 2 \pi) e^{-\left(x^{2}+y^{2}\right) / 2}$ be the normal density function, and $\varphi(r)=(1 / 2 \pi) e^{-r^{2} / 2}$ be its polar version. Let $b \sim(2 \log n)^{1 / 2}$ be the solution to $(1 / b) e^{-b^{2} / 2}=1 / n$, and let $p_{h}=\int_{-\infty}^{\infty} \int_{b}^{\infty} \varphi(x, y) d x d y$ be the measure of the halfplane to the right of the line $x=b$. It is important that $p_{h}$ be quite small.

Claim 2. $p_{h}=O(1 / n)$.

Proof. Let $z=(2 \log n)^{1 / 2}$. We can ignore those points at a distance more than $z$ from the origin, since their total measure is $\int_{z}^{\infty} 2 \pi r \varphi(r) d r=1 / n$. The remainder $p_{h}^{\prime}$ of $p_{h}$ can be expressed as an integral in polar coordinates as $p_{h}^{\prime}<\int_{b}^{z} 2 r \theta \varphi(r) d r$, where $\theta=\cos ^{-1}(b / r)$. For $r<z$ and $n$ large, $\theta$ is small. Let $t=r^{2} / b^{2}=1 / \cos ^{2} \theta>$ $1+\theta^{2}$. The integral becomes

$$
p_{h}^{\prime}<\frac{b^{2}}{2 \pi} \int_{1}^{\infty} e^{-b^{2} t / 2}(t-1)^{1 / 2} d t .
$$

But $b(t-1)^{1 / 2} \leq k$ for $t \leq 1+k^{2} / b^{2}$, so

$$
\begin{aligned}
p_{h}^{\prime} & <\sum_{k \geq 1} \frac{b k}{2 \pi} \int_{1+(k-1)^{2} / b^{2}}^{1+k^{2} / b^{2}} e^{-b^{2} t / 2} d t \\
& <\frac{1}{b \pi} e^{-b^{2} / 2} \sum_{k \geq 1} k e^{-(k-1)^{2} / 2} \\
& =\frac{c}{b} e^{-b^{2} / 2}
\end{aligned}
$$

for some positive constant $c$. That establishes the claim.

Now consider a circle $C$, centered at the origin, of radius $R=b+1 / b$. Those parts of the interior of $C$ which lie to the right of the line $x=b$ and to the left of line $x=-b$ will play the role of an ear, as in the preceding proof. Let $p_{\text {ear }}$ be the measure of an ear.

Claim 3. $p_{\text {ear }}=\Omega(1 / n)$.

Proof. On the circle of radius $R$, the probability density is $\varphi(R)>$ $(1 / 2 \pi) e^{-b^{2} / 2-2}=\Theta(b / n)$, by the choice of $b$, and the density over the ear is greater than that. The ear subtends an angle of $\theta \sim \sqrt{2} / b$, so the ear has area $\sim \theta^{3} R^{2} / 12=\Theta(1 / b)$, and measure at least $\left(\theta^{3} R^{2} / 12\right) \varphi(R)=\Omega(1 / n)$. 
Now choose $m$ to satisfy $2 \pi / m=\theta \sim \sqrt{2} / b$, which implies that $m=\Theta\left(\log ^{1 / 2} n\right)$. Round $m$ to the nearest even integer.

The remainder of the proof is almost identical to that of Theorem 1 . If the two ears under consideration are aligned so that their chords are vertical, then in addition to the previous conditions, a bad set of points must have no points to the right of the line $x=b$, or to the left of the line $x=-b$, so that the points in the green, yellow, and blue regions must be on the convex hull. By Claim 2, that happens with constant positive probability. The previous proof assumed that the probability density was uniform. Here, the density varies only by a constant factor over an ear, which is sufficient for the proof to carry through. The blue, green, and yellow regions occupy the same fraction of the ear as before, and have measure $\Omega\left(1 / n k^{3}\right)$, by Claim 3 .

As before, the result is that the expected modality is $\Omega(\log m / \log \log m)$. However, in this case that is $\Omega(\log \log n / \log \log \log n)$.

\section{Upper Bounds on Expected Modality}

Theorem 3. When $n$ points are drawn at random uniformly from a disk in the plane, the expected modality of their convex hull is $O(\log n / \log \log n)$.

Proof. The proof is similar in spirit to that of the lower bound; it shows that the lower bound construction is nearly optimal. Let $m=\left(\pi^{2} n / 4 \log n\right)^{1 / 3}$ and $\alpha=2 \pi / m$, and assume that the disk has radius 1 . An $\alpha$-ear is a region of the disk outside some chord subtending angle $\alpha$. Figure 5(a) shows an $\alpha$-ear, to the right of line $l$.

Claim 4. With probability approaching 1 as $n \rightarrow \infty$, no $\alpha$-ear is empty.

Proof. If any $\alpha$-ear is empty, then one of the ears associated with some fixed inscribed regular $2 m$-gon must also be empty. Each ear associated with a $2 m$-gon has measure $\sim(\alpha / 2)^{2} / 12 \pi \sim \log n / 3 n$. So the probability that any $\alpha$-ear is empty is asymptotically less than $2 m(1-\log n / 3 n)^{n}<2 m e^{-\log n / 3}=\left(2 \pi^{2} / \log n\right)^{1 / 3}$, which approaches 0 as $n \rightarrow \infty$.

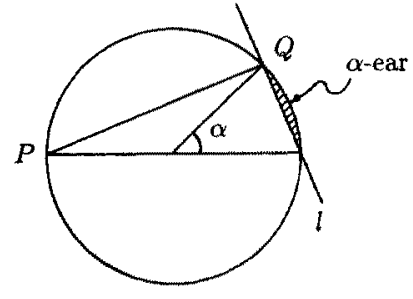

(a)

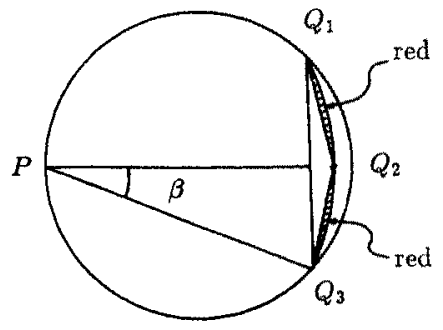

(b)

Fig. 5. The red regions. 
We presume that no $\alpha$-ear is empty. Hence, every hull point lies within a band between circles of radius $R=\cos (\alpha / 2)$ and radius 1 . Let $p_{\text {band }}=1-R^{2} \sim \alpha^{2} / 4=$ $\Theta\left(\log ^{2 / 3} n / n^{2 / 3}\right)$ be the measure of that band.

Let $P$ be an arbitrary hull point. Let $Q$ be another hull point which is a local maximum for $P$, and let $l$ be the line through $Q$ perpendicular to line $\overline{P Q}$, as shown in Fig. 5(a). In the figure, $P$ is shown to lie on the boundary, which for the present purposes is accurate. The part of the disk on the opposite side of $l$ from $P$ must be empty, since otherwise $Q$ could not be a local maximum for $P$. So if $Q$ does not lie in the $2 \alpha$-ear directly opposite $P$, then there must be an empty $\alpha$-ear, as shown in Fig. $5(\mathrm{a})$, which is presumed not the case. The measure of a $2 \alpha$-ear is $p_{\text {ear }} \sim(2 \alpha)^{3} / 12=\Theta(\log n / n)$.

Suppose a point $P$ has been chosen, along with $k$ other points $Q_{1}, \ldots, Q_{k}$. Those $k$ points can only be local maxima for $P$ if there is a local minimum hull point between adjacent pairs of local maxima. The probability of getting the necessary local minima is maximized when $Q_{1}, \ldots, Q_{k}$ lie equally spaced on an arc $A$ centered at $P$, as shown in Fig. 5(b) for $k=3$. Between each adjacent pair $Q_{i}$ and $Q_{i+1}$ is a red region, where a local minimum point for $P$ should lie. Since $Q_{1}, \ldots, Q_{k}$ all lie in the $2 \alpha$-ear opposite $P$, and $P$ is near the boundary, the angle $\beta$ at $P$ subtended by the portion of arc $A$ between adjacent local maxima is $\beta \sim \alpha /(k-1)$, and the area of each red region is $\sim \beta^{3} / 3$. The measure of each red region is thus $p_{\min } \sim \beta^{3} / 3 \pi=\Theta\left(m^{-3}(k-1)^{-3}\right)=\Theta\left(\log n / n k^{3}\right)$. Considering all possibilities, the probability $p_{k}$ that, for some hull point $P$, there are points $Q_{1}, \ldots, Q_{k}$ in the opposite $2 \alpha$-ear, with a local minimum between each adjacent pair, satisfies

$$
\begin{aligned}
p_{k} & <n p_{\text {band }}\left(\begin{array}{c}
n-1 \\
k
\end{array}\right) p_{\text {ear }}^{k}(n-k-1)^{k-1} p_{\min }^{k-1} \\
& <\frac{c^{k} n^{1 / 3}(\log n)^{2 k}}{(k-1)^{4 k-3}}
\end{aligned}
$$

for some constant $c>0$. Choosing $k=c^{\prime} \log n / \log \log n$ for $c^{\prime}>\frac{1}{6}$ causes $p_{k}$ to approach 0 as $n \rightarrow \infty$, and to do so at a superexponential rate in $k$. It follows that the expected modality is $O(\log n / \log \log n)$.

Again, the proof for points drawn from a normal distribution is very similar to the case of uniformly distributed points in a disk.

Theorem 4. When $n$ points are drawn at random from a two-dimensional normal distribution, the expected modality of their convex hull is $O(\log \log n / \log \log \log n)$.

Proof. This proof is similar to the preceding one, but is complicated slightly by the fact that the probability density is no longer uniform over an ear. Let $\lambda=2 \pi e \log ^{1 / 2} n \log \log n$ and $R=(2 \log (n / \lambda))^{1 / 2}$. Let $C$ be the circle of radius 


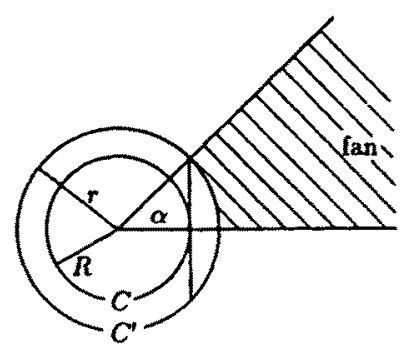

(a)

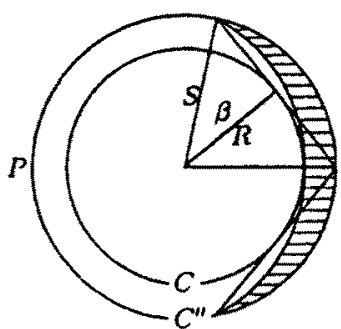

(b)

Fig. 6. The ears for Theorem 4.

$R$, centered at the origin. An $R$-half-plane is any half-plane not including the origin whose boundary line is tangent to circle $C$.

Claim 5. With probability approaching 1 as $n \rightarrow \infty$, no $R$-half-plane is empty.

Proof. Let $r=(2 \log (n / \lambda)+2)^{1 / 2}$, and let $C^{\prime}$ be a circle of radius $r$, concentric with $C$. Let $\alpha$ be the angle subtended by a chord of circle $C^{\prime}$, tangent to circle $C$, as shown in Fig. 6(a). One of $2 \pi / \alpha$ fans is shaded in Fig. 6(a). If any $R$-half-plane is empty, then one of those fans is empty. Note that $\cos \alpha=R / r \approx$ $1-1 / 2(\log n-\log \lambda)$, from which it follows that $\alpha \sim 1 / \log ^{1 / 2} n$. The measure of the region outside circle $C^{\prime}$ is $\int_{r}^{\infty} 2 \pi s \varphi(s) d s=e^{-r^{2} / 2}=\lambda / e n$. So the measure of each fan is $\alpha \lambda / 2 \pi e n$, and the probability that any fan is empty is less than $(2 \pi / \alpha)(1-\alpha \lambda / 2 \pi e n)^{n}<(2 \pi / \alpha) e^{-\alpha \lambda / 2 \pi e}$, which approaches 0 as $n \rightarrow \infty$.

Claim 6. With probability approaching 1 as $n \rightarrow \infty$, all hull points lie in a band whose boundaries are circles $C$ and $C^{\prime \prime}$, where $C^{\prime \prime}$ has radius $S=(2 \log (\lambda n))^{1 / 2}$, and is concentric with $C$. That band has measure $p_{\text {band }}<e^{-R^{2} / 2}=\lambda / n$.

Proof. If any hull point lies inside circle $C$, then some $R$-half-plane must be empty, which is presumed not the case. The probability that any point at all lies outside circle $C^{\prime \prime}$ is $<n e^{-S^{2} / 2}=1 / \lambda$, which approaches 0 as $n \rightarrow \infty$.

Let $P$ be an arbitrary hull point, without loss of generality on the $x$-axis, and let $Q$ be a local maximum hull point for $P$. Then $Q$ must lie within the shaded area of Fig. 6(b), which we call an ear. The ear consists of all points $Q$ in the band such that a line through $Q$, perpendicular to $\overline{P Q}$, does not intersect circle $C$. Otherwise, either $Q$ could not be a local maximum for $P$, or there would be an empty $R$-half-plane. As illustrated, the angle subtended by the ear is $4 \beta$. Approximately solving $\cos \beta=R / S=((\log n-\log \lambda) /(\log n+\log \lambda))^{1 / 2}$ gives $\beta \sim(2 \log \lambda / \log n)^{1 / 2}$. 
Claim 7. The measure of an ear is $p_{\mathrm{ear}}=O\left(\lambda / n \log ^{1 / 2} n\right)$.

Proof. At an angle of $\alpha$ above horizontal, the inner boundary of the ear is at a distance of $R+x$ from the origin, where $R /(R+x)=\cos \theta$ and $\theta \sim \alpha / 2$, from which it follows that $x>R \alpha^{2} / 10$. So

$$
\begin{aligned}
p_{\text {ear }} & <2 \int_{0}^{2 \beta} \int_{R+x}^{\infty} \frac{r}{2 \pi} e^{-r^{2} / 2} d r d \alpha \\
& <\frac{1}{\pi} \int_{0}^{2 \beta} e^{-(R+x)^{2} / 2} d \alpha \\
& <\frac{\lambda}{2 \pi n} \int_{0}^{\infty} e^{-R^{2} \alpha^{2} / 10} d \alpha \\
& <\frac{1}{\pi} e^{-R^{2} / 2} \int_{0}^{\infty} e^{-S^{2} \alpha^{2} / 10} d \alpha \\
& =\frac{\lambda \sqrt{10 \pi}}{2 \pi n R} \\
& =O\left(\frac{\lambda}{n \log ^{1 / 2} n}\right) .
\end{aligned}
$$

Now we give something away. Suppose that the probability density is uniform over the ear, taking on a value of $e^{-R^{2} / 2}=\lambda / n$. Then the best way to place $k$ points in the ear, in the hope that they might be local maxima for $P$, is to distribute them evenly on an arc centered on $P$. Assuming that the arc can span the entire length of the ear, which is optimistic, each region where a local minimum hull point must fall has measure $p_{\min } \sim(2 \beta /(k-1))^{3}\left(4 R^{2} / 12\right)(\lambda / n)$. Substituting $\beta \sim$ $(2 \log \lambda / \log n)^{1 / 2}, \lambda=2 \pi e \log ^{1 / 2} n \log \log n$ and $R \sim(2 \log n)^{1 / 2}$ gives $p_{\min }=$ $O\left((\log \log n)^{5 / 2} / n(k-1)^{3}\right)$. As in the case of a uniform distribution, the probability $p_{k}$ that the modality is at least $k$ satisfies

$$
\begin{gathered}
p_{k}<n p_{\text {band }}\left(\begin{array}{c}
n-1 \\
k
\end{array}\right) p_{\text {ear }}^{k}(n-k-1)^{k-1} p_{\min }^{k-1} \\
<\frac{(\log n)^{1 / 2} c^{k}(\log \log n)^{3.5 k-1.5}}{(k-1)^{4 k-3}}
\end{gathered}
$$

for some constant $c$. But $p_{k} \rightarrow 0$ as $n \rightarrow \infty$ provided $k \geq a \log \log n / \log \log \log n$, and $a>1$. Moreover, $p_{k}$ decreases superexponentially in $k$, so the expected value of $k$ is $O(\log \log n / \log \log \log n)$, and Theorem 4 is established.

\section{References}

1. A. Aggarwal, M. Klawe, S. Moran, D. Shor, and R. Wilber. Geometric applications of a matrix searching algorithm. In Proc. 2nd ACM Symposium on Computational Geometry, pp. 285-292, 1986. 
2. A. Aggarwal and R. C. Melville. Fast computation of the modality of polygons. J. Algorithms, 7:369-381, 1986.

3. D. Avis, G. T. Toussaint, and B. K. Bhattacharya. On the multimodality of distance in convex polygons. Comput. Math. Appl., 8(2):153-156, 1982.

4. D. P. Dobkin and L. Snyder. On a general method for maximizing and minimizing among geometric problems. In Proc. 20th Annual Symposium on Foundations of Computer Science, pp. 7-19, 1979.

5. F. P. Preparata and M. I. Shamos. Computational Geometry. Springer-Verlag, New York, 1985.

6. H. Raynaud, Sur l'enveloppe convexe des nuages des points aléatoires dans $R^{\prime \prime}$. J. Appl. Probab., $7: 35-58,1970$.

7. W. E. Snyder and D. A. Tang. Finding the extrema of a region. IEEE Trans. Pattern Anal. Mach. Intell, $2: 266-269,1980$.

8. G. T. Toussaint. Complexity, convexity, and unimodality. Internat. J. Comput. Inform. Sci., 13(3):197-217, 1984

Received July 7, 1987, and in revised form July 11, 1988. 